Editorial

\title{
Acknowledgment to Reviewers of $J$ in 2021
}

\section{$J$ Editorial Office}

Citation: J Editorial Office.

Acknowledgment to Reviewers of $J$

in 2021. J 2022, 5, 105-106.

https://doi.org/10.3390/j5010007

Published: 28 January 2022

Publisher's Note: MDPI stays neutral with regard to jurisdictional claims in published maps and institutional affiliations.

Copyright: (C) 2022 by the author. Licensee MDPI, Basel, Switzerland. This article is an open access article distributed under the terms and conditions of the Creative Commons Attribution (CC BY) license (https://creativecommons.org/licenses/by/4.0/).
MDPI AG, St. Alban-Anlage 66, 4052 Basel, Switzerland

Rigorous peer-reviews are the basis of high-quality academic publishing. Thanks to the great efforts of our reviewers, $J$ was able to maintain its standards for the high quality of its published papers. Thanks to the contribution of our reviewers, in 2021, the median time to first decision was 13 days and the median time to publication was 34 days. The editors would like to extend their gratitude and recognition to the following reviewers for their precious time and dedication, regardless of whether the papers they reviewed were finally published:

\begin{tabular}{|c|}
\hline Abelmann, Leon \\
\hline Amin, Farhan \\
\hline Andronov, Ivan V. \\
\hline Arvanitis, Konstantinos \\
\hline Auad, Lígia Isoni \\
\hline Beliatis, Michail J. \\
\hline Beňová, Mariana \\
\hline Bombino, Giuseppe \\
\hline Botelho, Raquel Braz Assuncao \\
\hline Botzheim, Janos \\
\hline Brzecka, Anna \\
\hline Bubola, Marijan \\
\hline Bukreev, Dmitriy A. \\
\hline Burrows, Katy \\
\hline Celakovska, Jarmila \\
\hline Chang, Kang-Ming \\
\hline Chęciński, Maciej \\
\hline Chileshe, Nicholas \\
\hline Chou, Pao-Nan \\
\hline Chukhrova, Nataliya \\
\hline Colombo-Mendoza, Luis Omar \\
\hline Corti, Fabio \\
\hline Dab̧rowska-Galas, Magdalena \\
\hline De Costa Trindade Amorim, Margarete Cristiane \\
\hline Dobrovolny, Hana M. \\
\hline Dos Santos, Demetrio Jackson \\
\hline Ejiro Naomi, Umaka \\
\hline Elshamy, Abdelsamed \\
\hline Faleiro, Eduardo \\
\hline Ferroni, Patrizia \\
\hline Fotia, Lidia \\
\hline Gao, Gary \\
\hline García-Giménez, Rosario \\
\hline Garus-Pakowska, Anna \\
\hline Genchi, Claudio \\
\hline Ghosh, Mrinmoy \\
\hline Godlewska, Joanna \\
\hline Gubar, Elena \\
\hline
\end{tabular}

Hadjisolomou, Ekaterini

Hallmann, Ewelina

Harata, Shinichiro

Hrynyszyn, Bozena Dorota

Ichim, Pavel

Imre, Attila R.

Inoue, Kohei

Jabłoński, Paweł

Janossy, Istvan

Jaworska, Beata

Kaiser, Norbert

Kang, Jeon-Young

Karmakar, Anwesa

Kenderessy, Pavol

Kitagawa, Jiro

Kochanowski, Maciej

Kozakiewicz, Marcin

Kozerozhets, Irina V.

Kumara, Indika

Kyzas, George Z.

Lascaris, Erik

Lawson, Aaron

Lelli, Francesco

Lerma, Claudia

Marcolin, Federica

Marques, Bruno

Marques, Cátia

Martynowicz, Helena

Maslov, Mikhail M.

Meng, Qingkai

Menghini, Luigi

Michał, Preisner

Michalowska, Joanna

Mognaschi, Maria Evelina

Musacchio, Massimo

Nadiruzzaman, Md.

Nara, Yasushi

Nemes, Norbert M. 
Netskina, Olga

Oancea, Bogdan

Ojovan, Michael I.

Okada, Hiroshi

Orynycz, Olga

Paluch, Andrew S.

Pant, Bishweshwar

Park, Hanbai

Perria, Rita

Prasadh, Somasundaram

Quadri, Marika

Quintas, Helder

Rasteiro, Deolinda M. L. Dias

Riccò, Matteo

Rodríguez López, María Isabel

Roy, Dipankar

Rubio, Cristina

Rybczyński, Maciej

Sato, Fuyuki

Scherrenberg, Martijn

Scholle, Markus

Sciolla, Jacopo Ciani

Setlak, Lucjan

Shah, Syyed Adnan Raheel

Sharma, Megha

Sher, Farooq
Shirvani Dastgerdi, Ahmadreza

Silva Filho, Edson

Silva, Marianne

Simo-Tagne, Merlin

Siódmiak, Jacek

Śliwa, Izabela

Soares Teles, Ariel

Staszczak, Magdalena

Stec, Piotr

Steffen, Claudius

Suso-Martí, Luis

Świątek, Łukasz

Talchabhadel, Rocky

Tamellin, Iacopo

Tauveron, Nicolas

Tostado-Véliz, Marcos

Trovato, Maria Rosa

Tsiakas, Konstantinos

Ulrich, Luca

Vieru, Dumitru

Vittori, Alessandro

White, Jeffrey

Yang, Hui-Ting

Yoshida, Norio

Zietkiewicz, Joanna 